\title{
Exploiting new generation ab initio and homology models from databases for MR
}

\author{
Adam J Simpkin ${ }^{1}$, Jens M H Thomas ${ }^{1}$, Ronan M Keegan², Daniel J Rigden ${ }^{1}$ \\ ${ }^{I}$ Institute of Integrative Biology, University of Liverpool, Liverpool L69 7ZB, England; \\ ${ }^{2}$ STFC, Rutherford Appleton Laboratory, Research Complex at Harwell, Didcot OX11 OFA, England \\ hlasimpk@liverpool.ac.uk
}

Molecular replacement (MR) is the primary method used to solve the phase problem in macromolecular crystallography. When there are no suitable homologues available for conventional MR, one alternative option is to predict the structure via bioinformatic means. This is referred to as ab initio or de novo modelling and has dramatically increased in accuracy in recent years with the availability of better residue-contact predictions derived through covariance analysis and deep learning. Covariance-assisted ab initio models are now available on a large scale in new generation databases, and therefore provide an easily obtainable source of potential search models as a supplement to the PDB. We have previously shown that using such structure predictions obtained from the GREMLIN and PconsFam databases could be processed with AMPLE to successfully solve structures through MR [1]. Here we explore the use of alternative model sources such as DMPfold and Alphafold2, considering which model preparation protocols are optimal.

We also present MrParse, a tool to access structures deposited to the PDB and predicted structures deposited in databases in a single, unified interface. This resource, which will also enable facile access to the latest automated homology models, will also present model quality assessment scores to help assess the quality of database-derived models. Presenting PDB structures and database-derived models in a common dashboard will help the crystallographer maximise the chance of success through MR.

[1] Simpkin et al. (2019) Acta Cryst D75(12) 1051-1062

Keywords: Molecular Replacement, Ab initio, MrParse, AlphaFold2 